\title{
Lower methane emissions were associated with higher abundance of ruminal Prevotella in a cohort of Colombian buffalos
}

Sandra Bibiana Aguilar-Marin ${ }^{1}$, Claudia Lorena Betancur-Murillo², Gustavo A. Isaza ${ }^{3}$, Henry Mesa ${ }^{1 *}$ and Juan Jovel ${ }^{4^{*}}$ iD

\begin{abstract}
Background: Ruminants burp massive amounts of methane into the atmosphere and significantly contribute to the deposition of greenhouse gases and the consequent global warming. It is therefore urgent to devise strategies to mitigate ruminant's methane emissions to alleviate climate change. Ruminal methanogenesis is accomplished by a series of methanogen archaea in the phylum Euryarchaeota, which piggyback into carbohydrate fermentation by utilizing residual hydrogen to produce methane. Abundance of methanogens, therefore, is expected to affect methane production. Furthermore, availability of hydrogen produced by cellulolytic bacteria acting upstream of methanogens is a rate-limiting factor for methane production. The aim of our study was to identify microbes associated with the production of methane which would constitute the basis for the design of mitigation strategies.
\end{abstract}

Results: Moderate differences in the abundance of methanogens were observed between groups. In addition, we present three lines of evidence suggesting an apparent higher abundance of a consortium of Prevotella species in animals with lower methane emissions. First, taxonomic classification revealed increased abundance of at least 29 species of Prevotella. Second, metagenome assembly identified increased abundance of Prevotella ruminicola and another species of Prevotella. Third, metabolic profiling of predicted proteins uncovered 25 enzymes with homology to Prevotella proteins more abundant in the low methane emissions group.

Conclusions: We propose that higher abundance of ruminal Prevotella increases the production of propionic acid and, in doing so, reduces the amount of hydrogen available for methanogenesis. However, further experimentation is required to ascertain the role of Prevotella on methane production and its potential to act as a methane production mitigator.

Keywords: Methane emissions, Methanogenesis, Prevotella abundance, Propionic acid synthesis

\footnotetext{
* Correspondence: Henry.mesa@ucaldas.edu.co; jovel@ualberta.ca

${ }^{1}$ Facultad de Ciencias Agropecuarias, Universidad de Caldas, Caldas, Colombia

${ }^{4}$ Office of Research. Faculty of Medicine and Dentistry, University of Alberta, Edmonton, AB T6G 2E1, Canada

Full list of author information is available at the end of the article
}

(c) The Author(s). 2020 Open Access This article is licensed under a Creative Commons Attribution 4.0 International License, which permits use, sharing, adaptation, distribution and reproduction in any medium or format, as long as you give appropriate credit to the original author(s) and the source, provide a link to the Creative Commons licence, and indicate if changes were made. The images or other third party material in this article are included in the article's Creative Commons. licence, unless indicated otherwise in a credit line to the material. If material is not included in the article's Creative Commons licence and your intended use is not permitted by statutory regulation or exceeds the permitted use, you will need to obtain permission directly from the copyright holder. To view a copy of this licence, visit http://creativecommons.org/licenses/by/4.0/ The Creative Commons Public Domain Dedication waiver (http://creativecommons.org/publicdomain/zero/1.0/) applies to the data made available in this article, unless otherwise stated in a credit line to the data. 


\section{Background}

Greenhouse gases include carbon dioxide $\left(\mathrm{CO}_{2}\right)$, methane $\left(\mathrm{CH}_{4}\right)$, nitric oxide $\left(\mathrm{N}_{2} \mathrm{O}\right)$ and ozone $\left(\mathrm{O}_{3}\right)$ [1]. The atmospheric content of $\mathrm{CO}_{2}$ and $\mathrm{CH}_{4}$ has increased dramatically since the industrial revolution [1] and are major contributors to global warming [2]. It has been estimated that methane may constitute up to $20 \%$ of greenhouse gases [3] and wetland and ruminant methane emissions have been on the rise since 2007, mainly in tropical and subtropical regions [4].

Livestock production systems may account for up to $14 \%$ of all anthropogenic methane emissions [5]. Consequently, more than 100 countries committed, in the Paris agreement of 2015, to reduce greenhouse emissions from agricultural activities [6]. In order to achieve this, however, a full understanding of methanogenesis and its associated microbes is needed. Methane is produced during enteric fermentation in ruminants by anaerobic microorganisms collectively known as methanogens in the Archaea domain and the phylum Euryarchaeota [7]. Plants fix atmospheric $\mathrm{CO}_{2}$ through photosynthesis and generate biomass rich in carbohydrates that is used to feed ruminants. Polysaccharides digestion then takes place under anoxic conditions in the rumen and hindgut [7] and includes a complex of anaerobic bacteria, fungi and protozoa that progressively process carbohydrates through hydrolysis and fermentation to produce acetic acid, $\mathrm{CO}_{2}$ and $\mathrm{H}_{2}$. Fermentation also generates short-chain fatty acids, like acetate, propionate and butyrate, which constitute an energy source for many cell metabolic processes and contribute to homeostasis of the digestive system [8]. Finally, methanogens convert $\mathrm{CO}_{2}$ and $\mathrm{H}_{2}$ to methane via the hydrogenotrophic pathway. Alternatively, the methylotrophic pathway produces methane using methylamines and methanol as substrates [9]. Methanogenesis is considered an essential process for ruminants because if hydrogen generated during carbohydrate fermentation is not removed, it may inhibit microbiome metabolism [7]. Finding ways to redirect hydrogen metabolism is a promising avenue to mitigate methane emissions and also to improve energy retention from grazing [10].

Changes in abundance of methanogens themselves would definitely affect methane production, but it remains also possible that changes in microbial composition and structure that result in perturbation of hydrogen metabolism or accumulation may also impact methanogenesis [11]. Indeed, application of candidate hydrogenotrophic bacteria that could redirect hydrogen away from methanogenesis has been proposed as a strategy to mitigate methane emissions [10]. More complex relationships are also possible. For example, it has been proposed that not only the abundance of methanogens but also the composition of methanogenic communities in the rumen seems to exert a strong effect on methane emissions. Namely, the presence of species within the Methanobrevibacter gottschalkii clade has been reported associated with higher production of methane [12]. Multiple factors including breed, sex and diet, affect microbiome composition [13, 14], and host genetics play a significant role in methane emissions without influencing microbiome composition [15].

The buffalo rumen microbiome remains largely underexplored. In preliminary studies, the rumen microbiome of Surti and Mehsani buffalos was found to be dominated by phylotypes belonging to the Bacteroidetes/ Chlorobi, Firmicutes and Protobacteria phyla, and the metagenome was consistent with a genetic profile specialized in carbohydrate fermentation [16]. Although in a very small cohort, Kala and collaborators reported that bacteria in the genera Prevotella, Bacteroides, Clostridium, Ruminococcus, Eubacterium, Parabacteroides, Fibrobacter and Butyrivibrio were the most abundant inhabitants of the buffalo rumen, and that the abundance of Ruminococcus flavefaciens and $R$. albus increased when animal were fed with high-roughage diet [17]. Finally, it was found that abundances of individual taxa and specific metabolites were correlated. For instance, Acetobacter abundance was positively correlated with acetate, propionate and butyrate content, and so was Prevotella abundance and butyrate content [18]. Thus, an important question that remains largely unanswered is how changes in microbiome structure affect methanogenesis. Clearly, more studies on the rumen microbiome in different breeds and geographical regions are urgently needed.

We conducted a microbiome survey in ruminal fluids of two cohorts of buffalos from a dairy farm in the department of Cordoba, Colombia, which were shown to produce low or high methane emissions. We hypothesized that animals with greater emissions of methane contained more abundant methanogenic microbes. However, our study revealed that the overall microbial composition did not appear very different among groups; instead, we detected higher abundance of Prevotella in the group with lower methane emissions. An hypothetical scenario to explain the inverse correlation between Prevotella abundance and methane emissions is presented.

\section{Results}

With the advent of next generation sequencing (NGS), the microbiome of ruminants is being profiled at high resolution and throughput and a complex picture is emerging wherein host genetics and microbiome structure additively contribute to several phenotypes, including methane emissions [13]. In order to investigate the ruminal microbiome composition of two cohorts of Colombian buffalos found to produce high or low levels of methane, we conducted 
shotgun metagenomics. The bioinformatics pipeline used in this study is described in Fig. 1.

\section{Alignment of individual sequences}

Taxonomic classification of sequences was performed using the software Kraken2 [19], and the standard database complemented with all bacterial, fungal, viral and archaeal sequences in the GenBank (including incomplete genomes) plus all sequences deposited in the Genome taxonomy database, GTDB (gtdb.ecogenomic.org). The GTDB hosts 145,512 bacterial accessions and 2392 archaeal accessions [20]. We discarded Kraken2 hits with less than $10 \%$ of the $\mathrm{k}$-mers matching the reference sequence. Then, we kept hits with a relative abundance of at least 3 reads (sequences) per sample. A list of hits obtained with Kraken2 pseudoalignments is presented in Supplementary Table S1. A total of 582 taxa were identified in our data. Five taxa corresponded to Archaea (Supplementary Fig. S1), along with 576 taxa in the domain Bacteria, one fungus of unknown taxonomy and two virus taxa of unknown taxonomy.

Principal coordinate analysis ordination of a BrayCurtis dissimilarity matrix showed that the microbiome of both groups of buffalos is apparently different, although there is considerable variability among animals in each group (Fig. 2a). The PCoA plot depicted in Fig. 2a shows that, along the first component (PC1), which capture $53 \%$ of the variance, most blue points (high methane emissions) located on the left part of the plot, while most red points (low methane emissions) clustered on the right part of the plot. Permutational Analysis of Variance (PERMANOVA), however, did not detect statistically significant differences between groups. We also conducted Analysis of Similarity (ANOSIM) and

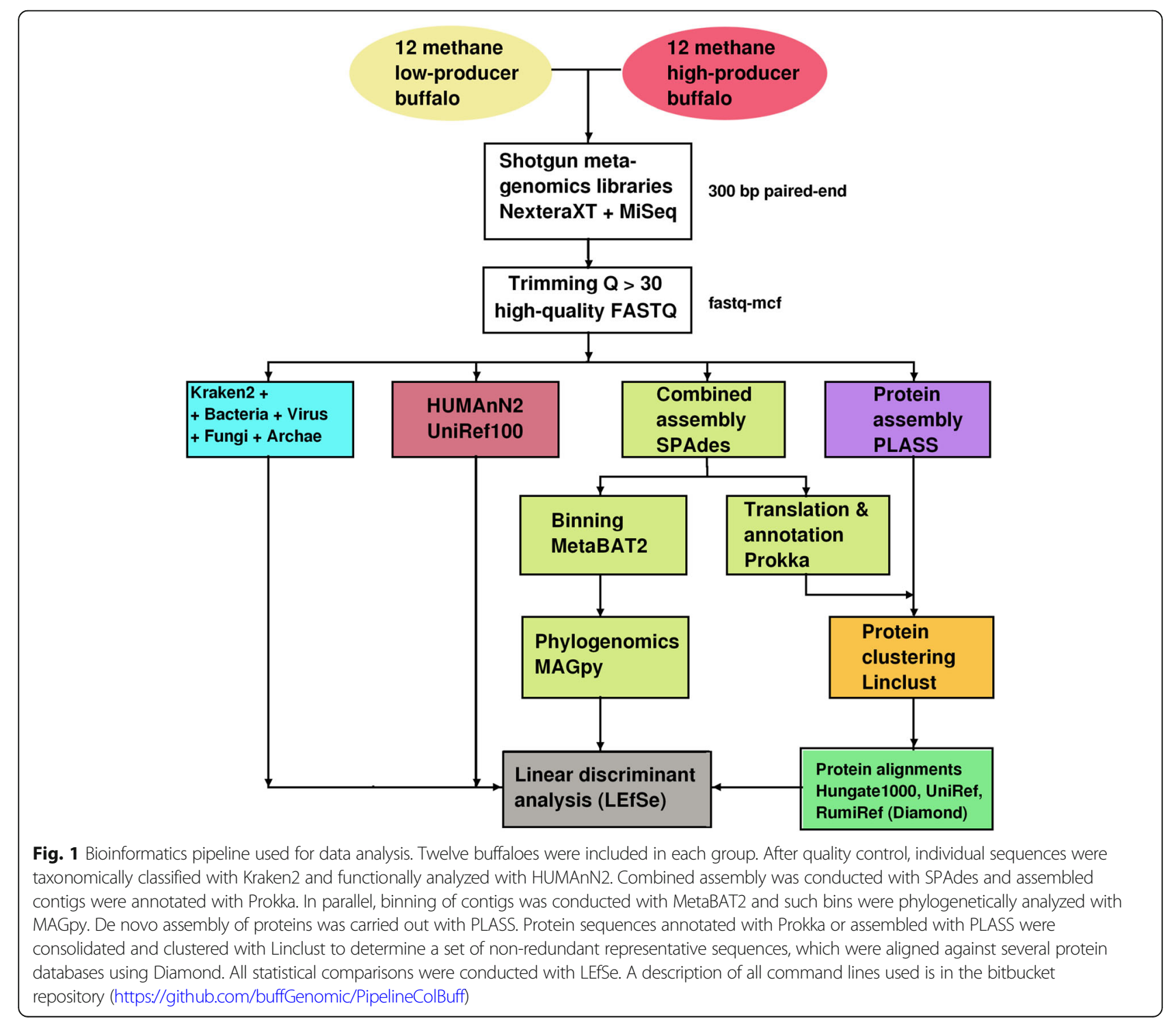






obtained a significant $p$-value (0.02), which again suggests that the groups under comparison are not statistically different. At the phylum level, the microbiome was dominated by Bacteroidetes and Firmicutes, and in decreasing abundance Actinobacteria and Protobacteria. Euryarchaeota was among the seven most abundant phyla, but with abundance much lower than that the rest of phyla (Fig. 2b). At the genus level, Prevotella was somewhat more abundant in the group of animals with lower methane emissions (Fig. 2e). At higher taxonomic levels, family, order and phylum, the same subtle trend was observed for Bacteroidaceae, Bacteroidales and Bacteroidota, respectively, which showed a moderately higher abundance in the group with lower methane emissions. However, high variability is evident inside each group.

We subjected the 100 most abundant taxa to hierarchical clustering of their Bray-Curtis dissimilarity indices using the hclust algorithm, which led to the identification of two clusters (Fig. 3). Namely, a larger cluster comprising 14 samples was integrated by 9 animals (64\%) from the low-methaneemissions group and 5 animals (36\%) from the highmethane-emissions group. This group exhibited higher abundance of the majority of the 100 most abundant bacteria, including 23 species of Prevotella (green bars), seven species of Butyrivibrio (blue bars), five species of Ruminococcus (red bars), also Selenomonas ruminantium, and Fibrobacter intestinalis, among others. The other cluster (on the 


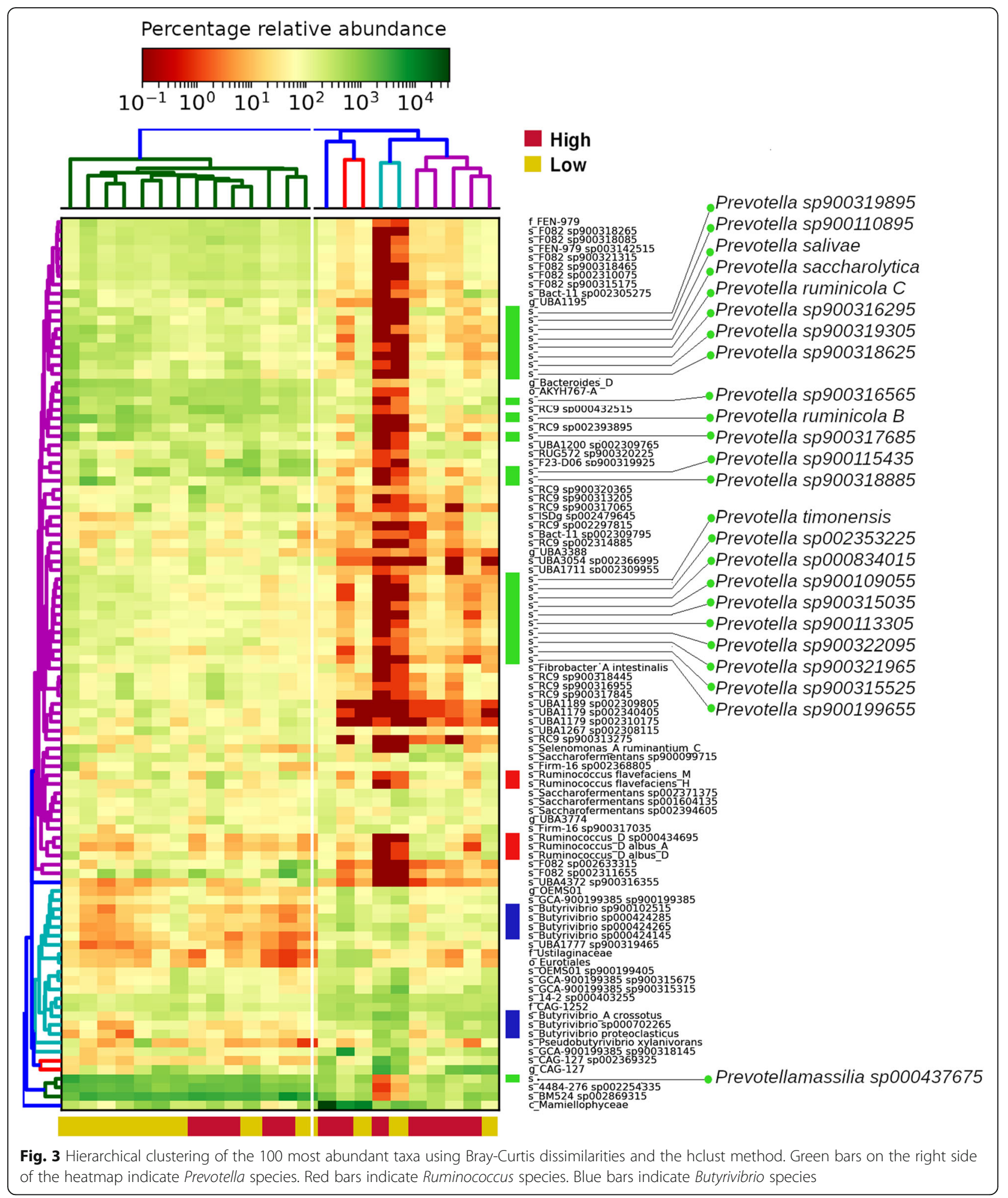

right), was more heterogeneous and contained mostly animals from the high-methane-emissions group.

We compared the abundance of the five taxa in the family Methanobacteriaceae detected in our libraries. In general, a subtle higher abundance in four out of five Methanobacteriaceae taxa in the group of animals with higher methane emissions was observed (Supplementary Fig. S1). Such differences did not reach statistical significance, but they are clearly 
appreciated in the boxplots, although considerable variability inside groups was also observed (Supplementary Fig. S1).

We subjected the abundance of all taxa detected to linear discriminant analysis, using the software LEfSe [21]. Interestingly, based on uncorrected $p$-values, LEfSe suggested that at least 29 species in the genus Prevotella were more abundant in the low-methaneemissions group (Fig. 4a). However, although LEfSe reported increased abundance of those Prevotella species, in most cases p-values lost significance after correction for multiple comparisons with the BenjaminiHochberg method. To better characterize this observation, we plotted the relative abundance of twelve species of Prevotella (Fig. 4b) and a clear trend is observed, although variability inside each group is considerably large.

We also conducted metabolic profiling with HUMAnN2 [22], but as is usual with non-human samples, results were incomplete and not very informative.

\section{Combined assembly and identification of bacterial} genomes

NGS libraries are a very fragmentary representation of the metagenome [23]. Thus, recovery of bacterial

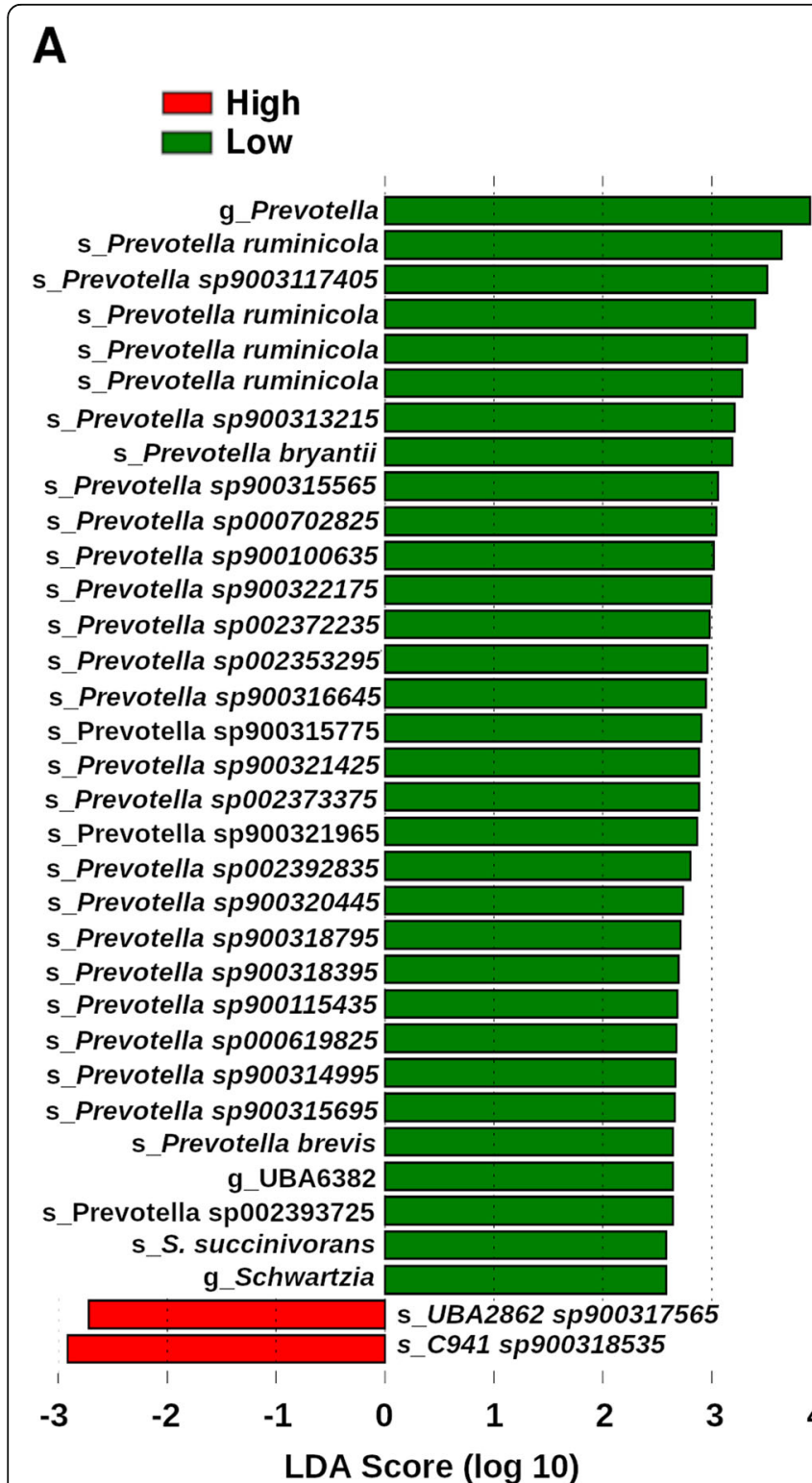

\section{B

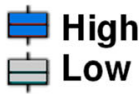

P. ruminicola $A$

P. ruminicola $B$

$P$ ruminicola $C$
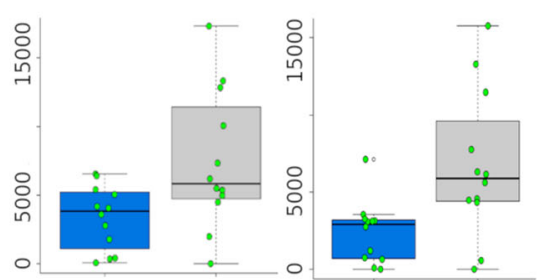

P. sp000702825

P. sp002372235

P. $\operatorname{sp} 900313215$
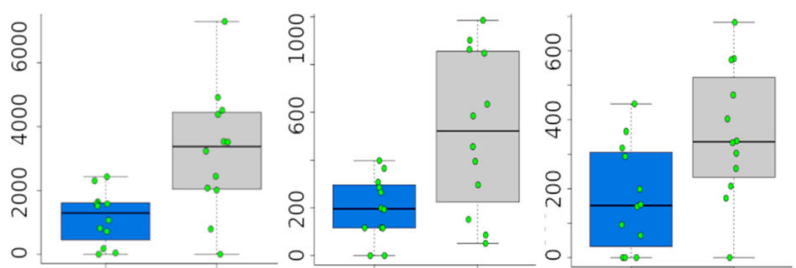

P. sp900315695

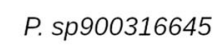

P. sp900315695
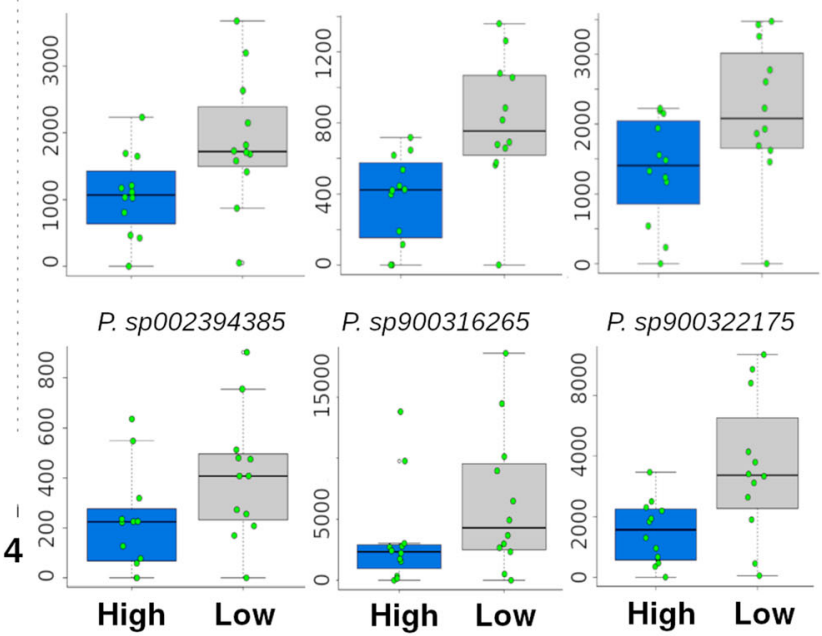

$\mathrm{CH}_{\mathbf{4}}$ emissions group

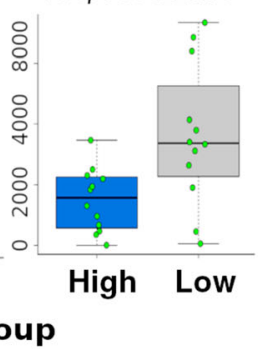

Fig. 4 Statistical comparison of taxa. a Linear discriminant analysis results. LEfSe was run with default parameters. b Box plots presenting the relative abundance of 12 Prevotella species in samples associated with high or low methane emissions 
genomes is incomplete and somewhat stochastic. This implies that in two or more samples harbouring the same bacterium, different parts of the genome might be recovered. Therefore, it makes sense to conduct sequence assembly combining all samples and after identification/annotation of assembled contigs, alignment of individual samples to assembled contigs will determine the relative contribution, if any, of each sample to each contig. Therefore we conducted combined assembly of all 24 samples. The assembly of reads generated 3.6 million contigs with an N50 of 481 bp (min. 373; max. 53, 776; average 491). We then subjected such contigs to binning with metaBAT2 [24]. From those, MetaBAT2 was able to cluster 78 putative bacterial genomes (bins), which were subjected to phylogenetics analysis and annotation with MAGpy [25]. Hereinafter, bins are referred to as MAGs.

The phylogenetic tree generated by MAGpy comprised three major clusters. The larger cluster (Fig. 5a; in black) contained many putative genomes that were very similar among them. The other two branches of the phylogenetic tree were more heterogeneous. The annotation assigned to each bin can be found in Supplementary Table S2. LEfSe analyses suggested that the genera Fibrobacter, Oscillibacter, Prevotella and the species Ruminococcaceae bacterium, Prevotella ruminicola, Bacteroidetes bacterium and a phage from Bacteroidetes were more abundant in the low-methane-emissions group, but $p$-values lost significance after correction for multiple comparisons. Because contigs used in this study are the result of an assembly procedure that included all samples, the relative contribution of each sample to each MAG is presented in Fig. 5b. Essentially, most MAGs were represented in all samples, with rather few exceptions (black cells in the lower half of heat map). The relative abundance of MAGs reported above is visibly larger in the group with lower methane emissions, but considerably heterogeneity is observed inside groups.

\section{Protein predictions and annotation}

We predicted a total of 4,467,657 representative nonredundant protein sequences that were aligned against the protein databases UniRef100 [26], RumiRef100 [26] and Hungate1000 [27]. RumiRef and Hungate1000 are databases of bacterial sequences from ruminal samples. Alignments were conducted with Diamond and the top hits were recovered. The best hit from each of the three alignments was selected for annotation.

To quantify the contribution of each sample to each protein sequence, individual samples sequences were aligned to the protein reference sequences with the method blastx of Diamond. An astringent filtering procedure was implemented as described in the Methods section. A total of 1309 protein sequences passed such filtering process (Supplementary Table S3). Among proteins most frequently detected were FAD-dependent oxidoreductase, Acyl-CoA dehydrogenase, Urocanate hydratase (homologous to Prevotella), subunit beta of a DNA-directed RNA polymerase, Methylmalonyl-CoA mutase, Pyridine nucleotide-disulfide oxidoreductase (homologous to $\mathrm{Eu}$ bacterium cellulosolvens) and a Glutamate formimidoyltransferase (homologous to Prevotella sp. HUN102), among many others (see Supplementary Table S3).

When we conducted hierarchical clustering on the 100 most abundant proteins, the exact same larger cluster of samples identified with Kraken2 taxonomy assignments (Fig. 3a) was recapitulated (Fig. 6a). Based on linear discriminant analysis, 60 enzymes were more abundant in the group with lower emissions of methane, and only 10 were more abundant in the group with higher emissions of methane. Interestingly, 25 enzymes with homology to Prevotella proteins are among the hits more abundant in the low-methane-emissions group (Fig. 6b). All those hits, however, were not statistically significant after correction for multiple comparison.

In summary, we conducted a thorough characterization of the rumen microbiome of two small cohorts of buffalos that were found to produce either high or low methane emissions. The main findings suggest increased abundance of Prevotella species in the group of low methane emissions. This was very clear in results from several analytical approaches presented here, however statistical significance was not reached in many cases, which probably is derived from intra-group variability. Lack of statistical significance in microbiome means comparisons is often the result of the large number of taxa detected, which makes correction of $p$-values very stringent. The number of animals in each of our experimental groups was relatively small $(n=12)$, which will also result in relatively high p-values. Nonetheless, and apparent higher abundance of Prevotella in the group of animals with lower methane emissions is evident.

\section{Discussion}

In this report we present the characterization of the microbiome of two cohorts of Colombian buffalos that produced either high or low methane emissions, in search for microbial determinants of methane production. Our original hypothesis was that a difference in methananogens abundance was the underlying cause of differential methane emissions. We did find subtle differences in four out of five Methanobacteriaceae taxa detected in our data. In all four cases, although considerable variability inside groups was evident, animals of the group associated with higher emissions exhibited higher abundance of such methanogens. So, it is possible that subtle differences in methanogens are sufficient to influence methane emissions. 


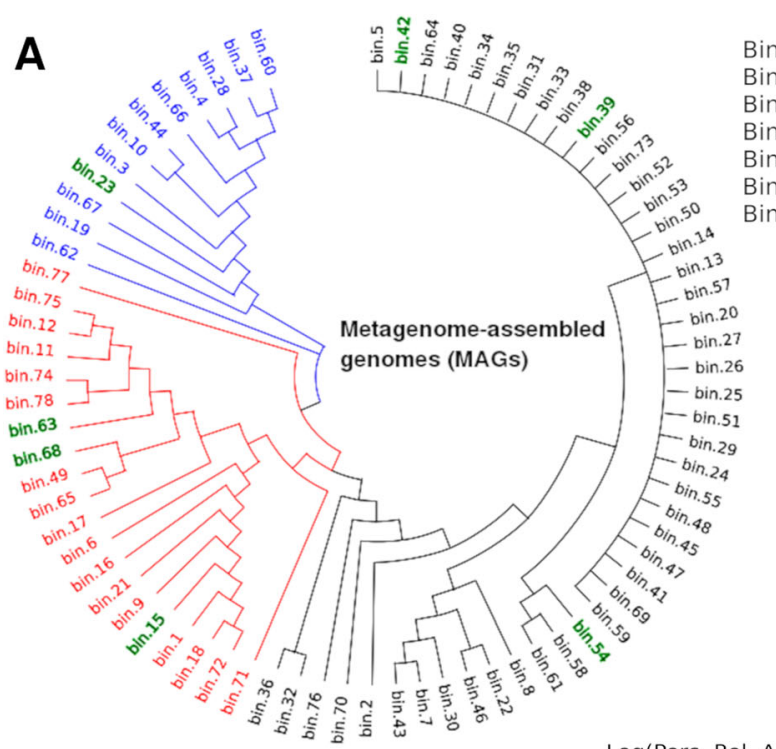

Bin23: Fibrobacter

Bin63: Oscillibacter

6in6: Rumminococcaceae bacterium

Bin15: Prevotella ruminicola

Bin54: Bacteroidetes bacterium

Bin39: Bacteroides phage

Bin42: Prevotella

B

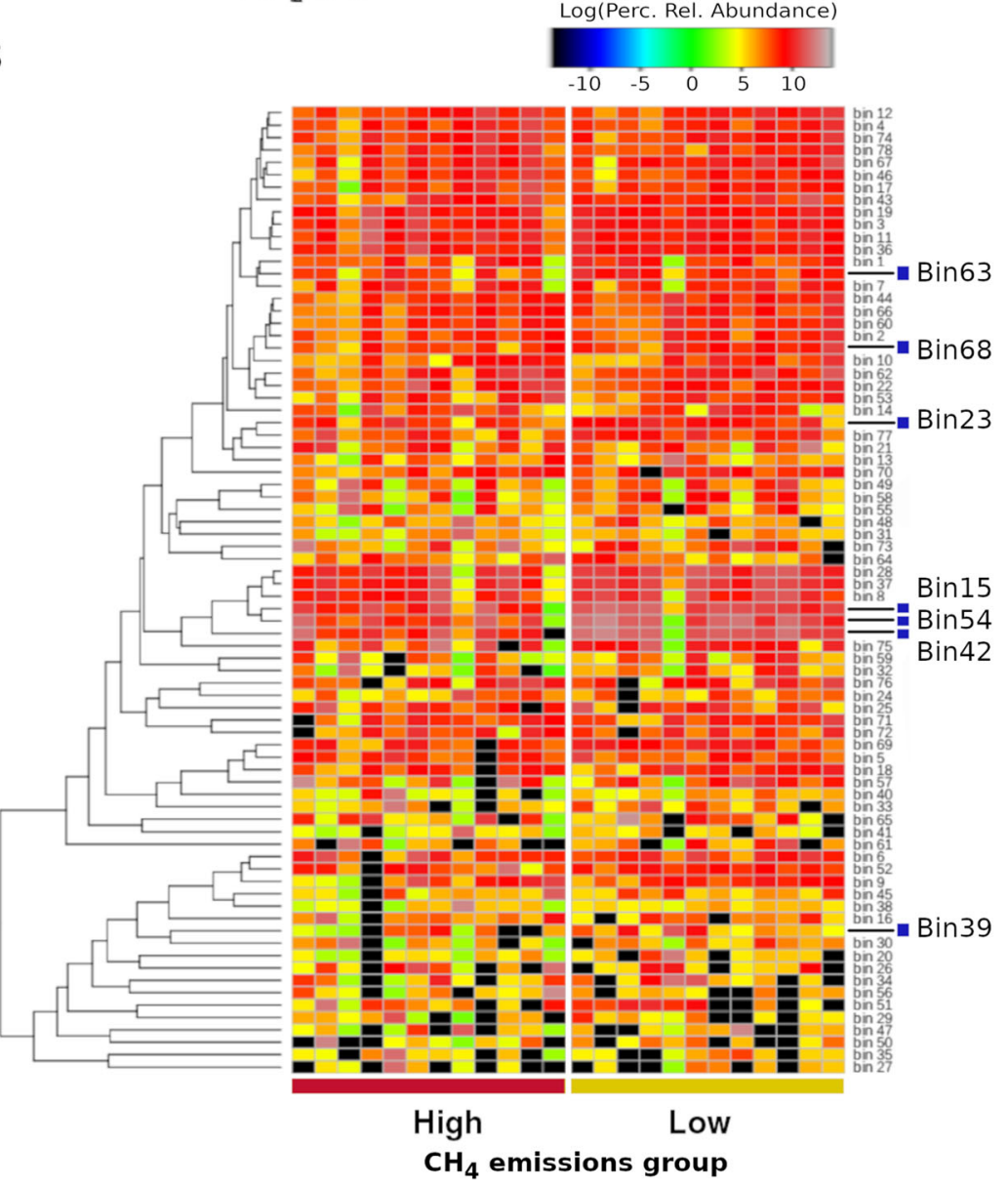

Fig. 5 Metagenome assembled genomes (MAGs). a Phylogenetic tree generated by MAGpy. b Relative contribution of each sample to each putative genome

More generally, and in agreement with previous reports, we found that the buffalos microbiome was mainly composed by Bacteroidetes and Firmicutes phyla [17, 18,
28]. At the family level, Bacteroidaceae, Lachnospiraceae and F082 predominated, while at the genus level, Prevotella and Butyrivibrio were dominant. High abundance 


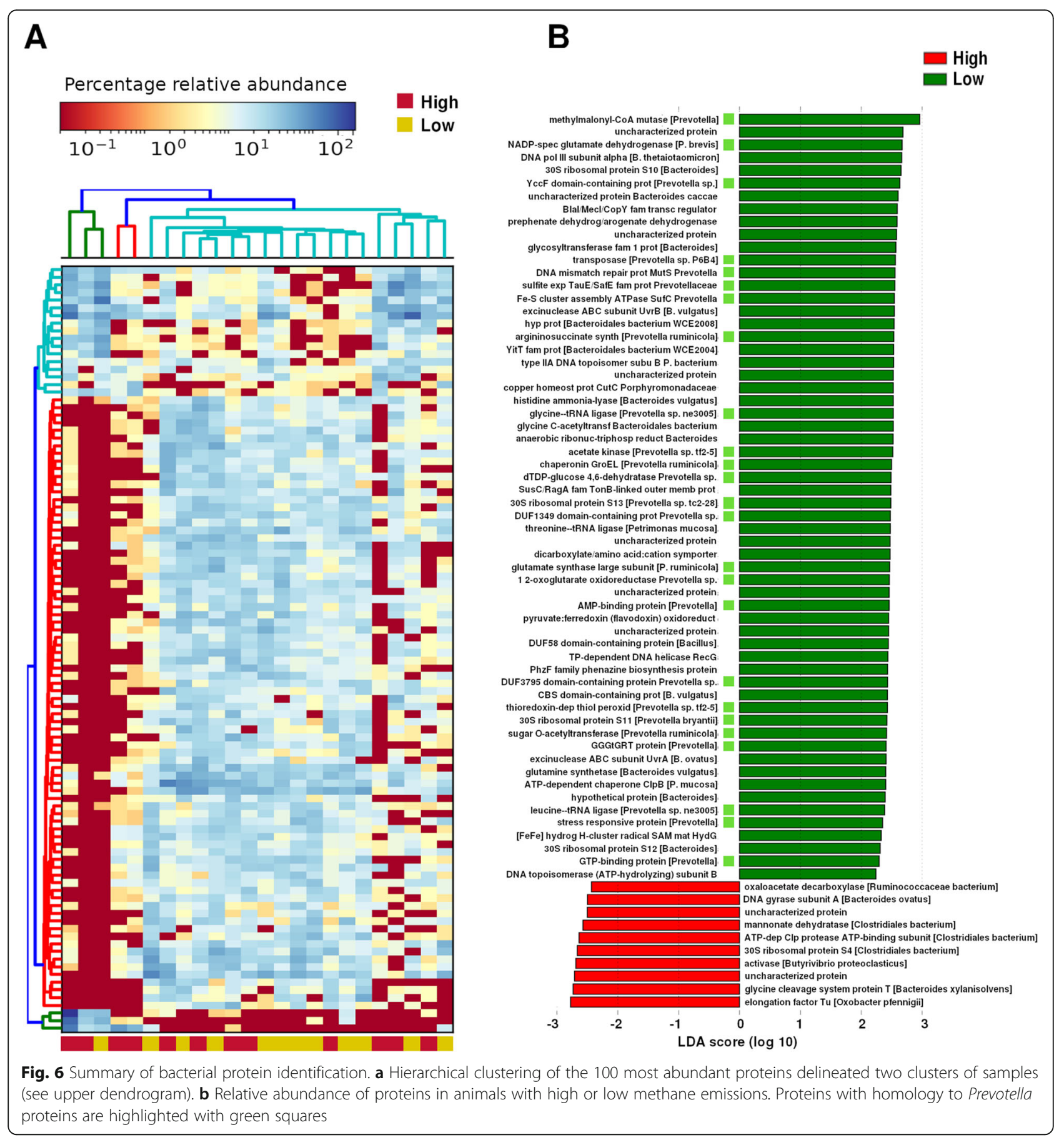

of Prevotella in the rumen microbiome has been reported by several groups in the past [29-33]. Importantly, at all taxonomic levels, the abundance of Prevotella, or the upper taxa containing it, were visibly more abundant in the group of animals with lower methane emissions. This effect was more pronounced at the genus level, where 10 out of 12 animal showed high proportion of Prevotella.
Taxonomic classification of sequences with Kraken2 [19] also suggested higher abundance of Prevotella in the group with lower methane emissions. This was confirmed by phylogenetic analyses with MAGpy [25]. This approach suggested that Prevotella ruminicola and another species in the genus Prevotella were more abundant in the group of lower methane emissions. Furthermore, after prediction an annotation of bacterial 
proteins, 60 enzymes were found to be more abundant in the group of lower methane emissions and from those 25 corresponded to protein sequences with high homology to Prevotella proteins. Thus, from these three lines of evidence we conclude that sequences with homology to Prevotella were more abundant in the group of animals with lower methane emissions. However, we do acknowledge that after correction of $p$-values for multiple comparisons, most comparisons were statistically nonsignificant, but the trend observed is very clear and favors the notion that Prevotella is more abundant in animals producing less methane. We want to point out that Prevotella is likely not the only cause of lower methane emissions and other factors, like animal genotype for example, might also exert an effect on such phenotype, as has been previously suggested [15].

The above-described observation poses the central question of this discussion. How can we explain reduction in methane emissions based on increased abundance of ruminal Prevotella? We favor the hypothesis that increase in the production of propionic acid by Prevotella reduces availability of hydrogen for methane production. In different contexts, it has been suggested that hydrogenotrophic bacteria might be used to divert hydrogen away from methane synthesis [10].

Diet of herbivores consists mostly of complex polysaccharides that are not digestible by host cells and most energetic requirements are satisfied through microbial fermentation [34]. In a simplified manner, polysaccharides breakdown starts with the adhesion of cellulolytic bacteria like Ruminococcus and Fibrobacter to the substrate. Solubilized polymers, formate, succinate, but also $\mathrm{CO}_{2}$ and $\mathrm{H}_{2}$, are then intercepted by butyrate-producing bacteria like Butyrivibrio and Roseburia and succinateand propionate-producing bacteria like Bacteroides and Prevotella. Methanogenic archaea compete for the hydrogen pool [34]. Experimentally, it has been demonstrated that adherent fibers and the liquid fraction of the rumen contain similar microbial ensembles, which vary in relative abundance, while the rumen epithelium harbor unique microbial taxa. Namely, adherent fibers are rich in fibrolytic microorganisms like member of the family Ruminococcaceae and the genus Fibrobacter, while the aqueous phase have abundant members of Prevotellaceae $[35,36]$. Interestingly, in our phylogenetic analyses with MAGpy we found increased abundance of Fibrobacter, Oscillibacter, Ruminococcaea bacterium, Prevotella, and Bacteriodetes bacterium in the group with lower methane emissions, which perhaps suggests a microbiome with higher fermentative capabilities.

Taxonomy of Prevotella ruminicola, one of the best studied species of Prevotella, has been revised several times considering microbiological and biochemical evidence. P. ruminicola was originally known as Bacteroides ruminicola [37]. As far back as 1966, it was demonstrated, using isotopic and enzymatic techniques, that $B$. ruminicola used the acrylate reductive pathway (using acrylyl-CoA as an intermediate) to produce propionate [38]. Not long after, Van Nevel and collaborators elegantly showed that inhibition of methanogenesis by chloral hydrate led to accumulation of gaseous hydrogen and an increase in propionic acid production. It is therefore accepted that metabolic hydrogen produced during ruminal fermentation is partitioned between production of methane, propionic acid and butyric acid [39].

\section{Conclusions}

We propose that higher abundance of Prevotella in the rumen of animals with lower methane emissions negatively influences methane production. More abundant Prevotella species would outcompete methanogens for hydrogen utilization, which will be diverted for production of propionic acid.

Of course, our hypothesis needs experimental validation. A simple way to test it would be to measure the content of propionate in animals with high or low abundance of Prevotella and methane emissions. Artificial enrichment of Prevotella from cultures is also an appealing approach to study metabolic flow. Ultimately, if proven true, microbiota from low methane emission animals could be transferred via ruminal liquid to animals with high production of methane and colonization of the rumen in the latter group would be monitored as well as methane emissions. Transferring the whole microbiome has the obvious advantage that any other microorganism contributing to the hypothesized role of Prevotella would also be transferred. Finally, we do not discard a role of genetic factors on methane production. Studies to characterize the genotype in animals with low or high methane production are currently underway.

\section{Methods}

Description of animals and collection of ruminal samples

The buffalo dairy farm is located at $8^{\circ} 10^{\prime} 34^{\prime \prime} \mathrm{N}$ and $76^{\circ} 03^{\prime} 46^{\prime \prime} \mathrm{W}$ in the Tierra Alta locality, in the department of Cordoba, Colombia. The dairy farm contains 11, 718 animals, and a database with records that extend back 22 years. For the measurement of $\mathrm{CH}_{4}$ emissions, four breathing chambers of metal structure with walls and roof covered with high-density polyethylene and sealed with velcro were used. Dimensions of each chamber were $2.2 \mathrm{~m} \times 1.7 \mathrm{~m} \times 0.9 \mathrm{~m}$ length, height and width, respectively, and allowed to control measurement conditions by confining the animals in small spaces and acting as flow accumulators of the gases belched and coming from the excreta. A Gases PRO Sensor Board (Calibrated) from Waspmote (Libelium ${ }^{\circ}$ ) was used, together with a Methane and Fuel Gas Sensor (Calibrated) 
reference $\mathrm{CH}-\mathrm{A} 3$, also from Libelium ${ }^{\circ}$. The sensor can perform $\mathrm{CH}_{4}$ measurements in the environment every $30 \mathrm{~s}$, with a nominal low explosive level (LEL) between zero and $100 \%$ with an accuracy of $\pm 0.15 \%$ LEL. One buffalo was placed in each of the chambers and the walls were sealed $5 \mathrm{~min}$ before the measurement corresponding to each chamber. The measurement was performed individually for each animal for a period of $10 \mathrm{~min}$ with captures of $\mathrm{CH}_{4}$ in the environment of the breathing chamber by the sensor every $30 \mathrm{~s}$, followed by a measurement of $\mathrm{CH}_{4}$ in the environment outside the chamber for a period of $5 \mathrm{~min}$ with the same sensor settings. In this way it was possible to obtain the difference between the $\mathrm{CH}_{4}$ concentration inside the chamber and the surrounding environment. In total 115 female and six male buffaloes were used to measure methane breath emissions. Each animal was measured three times a day, each measurement lasting $10 \mathrm{~min}$.

Twelve animals, which were found to emit either high $\left(1.36 \pm 0.11 \mathrm{~g} \mathrm{CH}_{4} / \mathrm{kg}\right.$ dry matter ingested) or low ($1.36 \pm 0.16 \mathrm{~g} \mathrm{CH}_{4} / \mathrm{kg}$ dry matter ingested) amounts of methane were included in each group.

\section{Sample collection, DNA extraction, library construction and sequencing}

For collection of ruminal samples, animals were sedated with Xylazine $(10 \%)$. The jaw of animals was immobilized and a $1 / 2$ in. siliconized probe was introduced and ruminal liquid was extracted using a manual Humboldt pump into sterile glass bottles. Samples were then aliquoted into $50 \mathrm{ml}$ Falcon tubes and snap-frozen in liquid nitrogen.

DNA was extracted using the QIAamp PowerFecal DNA Kit (QIAGEN) according to manufacturer's instructions, which includes bead-beating. DNA was then quantified using Qubit and a dsDNA HS Assay kit (Thermo Fisher Scientific). NexteraXT (Illumina) libraries were constructed from 1 ng of genomic DNA according to manufacturer's protocols. Indexed libraries were then inspected on a high sensitivity Bioanalyzer 2000 chip (Agilent) and quantified using Qubit as above. For library pooling, molarity of libraries was calculated using the average library size and the DNA concentration and a $4 \mathrm{nM}$ pool was prepared. Libraries were sequenced at $10 \mathrm{pM}$ on a MiSeq instrument (Illumina) using a $300 \mathrm{cy}-$ cles paired-end protocol that included demultiplexing.

\section{Bioinformatics analysis}

Sequence's quality was inspected with fastqc and bases with $\mathrm{Q}$ scores $<30$ were trimmed off with fastq-mcf keeping only sequences with a final length $>100$ bases. These are referred to as 'clean-sequences' and were used for all downstream procedures.

\section{Taxonomic classification}

Sequences were classified using Kraken2 [19]. We used the Kraken standard database complemented with all whole-genome and partial sequences of bacteria, archaea, fungi and viruses found in NCBI. Such database was complemented with the Genome Taxonomy Database, GTDB [20] (Fig. 1). Kraken2 results were filtered to allow only hits with at least $10 \% \mathrm{k}$-mers aligned.

\section{Combined assembly}

We conducted combined assembly of all our 24 samples with SPAdes [40], with default parameters. For deconvolution of assembled contigs, each individual sample was mapped to each contig and the number of aligned reads to each contig was normalized by library size and was considered the relative contribution of each sample to each contig.

\section{Metagenome-assembled genome prediction}

Contigs generated with SPAdes were also subjected to phylogenetic analysis with MAGpy [25]. Initially, contigs were binned with MetaBAT2 [24] and such bins were then analyzed with MAGpy. MetaBAT2 generates a file where the relative contribution of each sample to each bin is indicated. We normalized such a table for comparisons of bins after MAGpy annotation.

\section{Protein sequences generation}

In order to maximize chances of detecting putative bacterial proteins, two complementary approaches were implemented. First, we annotated in silico translated contigs from combined assembly with the software Prokka [41]. Prokka utilizes third-party feature prediction tools to identify genomic features contained in contigs. It translates in silico the features identified and annotates them by comparison with bacterial protein databases. One of the outputs is a FASTA file containing protein sequences predicted from the scaffolds. Second, we used PLASS [42] to assemble protein sequences de novo. See Fig. 1 for a schematic of our workflow. Since the Prokka translated and the PLASS predicted protein sets may be partially redundant, we concatenated them and conducted clustering with Linclust [43] to obtain a non-redundant set of representative sequences.

\section{Alignments of protein against several databases}

We conducted Diamond [44] alignments against the database UniRef [26] and against two databases containing ruminal bacterial proteins, UniRef [29] and Hungate1000 [27]. Because RumiRef includes annotations derived from alignments against multiple databases (CAZy, KEGG, UniRef and Hungate) when possible, we recovered all annotations. To determine the relative contribution of each sample to each protein sequence, each 
library was aligned with Diamond (method blastx) against the database of non-redundant protein representative sequences and to account for the resolution lost during clustering of protein sequences, a hit was considered true if it had an identity of at least $90 \%$ over a stretch of at least 50 amino acids. Moreover, we discarded hits for which less than five reads per sample, on average, were registered and hits that were present in less than three samples in each group.

\section{Statistical analysis}

In all cases, statistical comparisons were conducted using linear discriminant analyses with the software LEfSe [21] or by conducting Wilcoxon test in R. A detailed workflow and required scripts describing the implementation of our analysis is publicly available in GitHub (https://github. com/buffGenomic/PipelineColBuff).

\section{Supplementary Information}

The online version contains supplementary material available at https://doi. org/10.1186/s12866-020-02037-6.

Additional file 1: Supplementary Figure S1. Relative abundance of

five Methanobacteriacea taxa identified.

Additional file 2: Supplementary Table S1. Results of taxonomic classification of sequences conducted with Kraken2.

Additional file 3: Supplementary Table S2. Annotation of MetaBat2 bins and relative abundance in each sample.

Additional file 4: Supplementary Table S3. Annotation and relative abundance of predicted proteins upon alignments against several protein databases.

\section{Abbreviations}

GTDB: Genome Taxonomy Database; NCBI: National Center for Biotechnology Information; MAGpy: A computational pipeline for the downstream analysis of Metagenome-Assembled Genomes (MAGs); LEL: Low Explosive Level; MTDFREML: Multiple-Trait Derivative -Free REstricted Maximum Likelihood; Qscore: Quality score; SPAdes: A genome assembly algorithm; MetaBAT2: Software to bin contigs; Prokka: Software for prokaryotic genome annotation; PLASS: Protein-Level ASSembler; UniRef: Comprehensive and non-redundant UniProt reference clusters; RumiRef: Reference database of protein clusters identified in the rumen microbiome; CAZy: Database of Carbohydrate-Active enZYmes.; KEGG: Kyoto Encyclopedia of Genes and Genomes; Blastx: An algorithm to search protein databases using a translated nucleotide query; LEfSe: Linear discriminant analysis Effect Size; GitHub: A portal that provides hosting for software development and version control using Git; NGS: Next Generation Sequencing; HUMANn2: HMP Unified Metabolic Analysis Network; SRA: Sequence Read Archive;

Hungate1000: Reference set of rumen microbial genome sequences

\section{Acknowledgements}

We would like to thank Jordan Patterson, for compiling the Kraken2 database and to Naomi Hotte and Heather Armstrong (University of Alberta) for critically reading a previous version of this manuscript. We thank the Programa de Doctorado en Ciencias Agrarias de la Facultad de Ciencias Agropecuarias de la Universidad de Caldas for their scientific and financial support.

\section{Ethics approval and consent participate}

All protocols were revised and approved by the Ethics Committee of the Universidad de Caldas.

\section{Authors' contributions}

SBAM, HME and JJ designed the study. SBAM, CLBM and HME collected samples. SBAM and CLBM processed samples. SBAM, GAIE and JJ analyzed data. All authors wrote and approved the manuscript.

\section{Funding}

This study was funded by Universidad de Caldas (Grants 0238018, http:// www.ucaldas.edu.co). The funders had no role in study design, data collection and analysis, decision to publish, or preparation of the manuscript.

\section{Availability of data and materials}

All sequences generated in this study are publicly available from the SRA portal of NCBI under the accession number PRJNA605425.

\section{Consent for publication}

Does not apply.

\section{Competing interests}

The authors have declared that no competing interests exist.

\section{Author details}

${ }^{1}$ Facultad de Ciencias Agropecuarias, Universidad de Caldas, Caldas, Colombia. Escuela de Ciencias Básicas, Tecnología e Ingeniería, Universidad Nacional Abierta y a Distancia, UNAD, Bogotá, Colombia. ${ }^{3}$ Departamento de Sistemas e Informática - Facultad de Ingenierías, Universidad de Caldas, Caldas, Colombia. ${ }^{4}$ Office of Research. Faculty of Medicine and Dentistry, University of Alberta, Edmonton, AB T6G 2E1, Canada.

Received: 7 July 2020 Accepted: 5 November 2020

Published online: 27 November 2020

\section{References}

1. Tian H, Lu C, Ciais P, Michalak AM, Canadell JG, Saikawa E, et al. The terrestrial biosphere as a net source of greenhouse gases to the atmosphere. Nature. 2016;531:225-8.

2. Huang J, Yu H, Dai A, Wei Y, Kang L. Drylands face potential threat under 2 ${ }^{\circ} \mathrm{C}$ global warming target. Nat Clim Chang. 2017;7:417-22.

3. Kirschke S, Bousquet P, Ciais P, Saunois M, Canadell JG, Dlugokencky EJ, et al. Three decades of global methane sources and sinks. Nat Geosci. 2013; 6:813-23.

4. Nisbet EG, Dlugokencky EJ, Bousquet P. Methane on the rise - again. Science. 2014;343:493-5.

5. Huws SA, Creevey CJ, Oyama LB, Mizrahi I, Denman SE, Popova M, et al. Addressing global ruminant agricultural challenges through understanding the rumen microbiome: Past, present, and future. Front Microbiol. 2018;9: 2161.

6. Wollenberg $E$, Richards M, Smith $\mathrm{P}$, Havlík $\mathrm{P}$, Obersteiner M, Tubiello FN, et al. Reducing emissions from agriculture to meet the $2{ }^{\circ} \mathrm{C}$ target. Glob Chang Biol. 2016;22:3859-64. https://doi.org/10.1111/gcb.13340.

7. Hook SE, Wright ADG, McBride BW. Methanogens: methane producers of the rumen and mitigation strategies. Archaea. 2010;2010.

8. Matthews C, Crispie F, Lewis E, Reid M, OToole PW, Cotter PD. The rumen microbiome: a crucial consideration when optimising milk and meat production and nitrogen utilisation efficiency. Gut Microbes. 2019;10:115-32.

9. Thauer RK, Kaster AK, Seedorf H, Buckel W, Hedderich R. Methanogenic archaea: ecologically relevant differences in energy conservation. Nat Rev Microbiol. 2008;6:579-91.

10. Lan W, Yang C. Ruminal methane production: associated microorganisms and the potential of applying hydrogen-utilizing bacteria for mitigation. Sci Total Environ. 2019;654:1270-83.

11. Söllinger A, Tveit AT, Poulsen M, Noel SJ, Bengtsson M, Bernhardt J, et al. Holistic Assessment of Rumen Microbiome Dynamics through Quantitative Metatranscriptomics Reveals Multifunctional Redundancy during Key Steps of Anaerobic Feed Degradation. mSystems. 2018;3.

12. Tapio I, Snelling TJ, Strozzi F, Wallace RJ. The ruminal microbiome associated with methane emissions from ruminant livestock. J Animal Sci Biotechnology. 2017;8.

13. John Wallace R, Sasson G, Garnsworthy PC, Tapio I, Gregson E, Bani P, et al. A heritable subset of the core rumen microbiome dictates dairy cow productivity and emissions. Sci Adv. 2019;5. 
14. Li F, Li C, Chen Y, Liu J, Zhang C, Irving B, et al. Host genetics influence the rumen microbiota and heritable rumen microbial features associate with feed efficiency in cattle. Microbiome. 2019;7.

15. Difford GF, Plichta DR, Løvendahl P, Lassen J, Noel SJ, Højberg O, et al. Host genetics and the rumen microbiome jointly associate with methane emissions in dairy cows. PLoS Genet. 2018;14:e1007580. https://doi.org/10. 1371/journal.pgen.1007580.

16. Singh KM, Reddy B, Patel AK, Panchasara H, Parmar N, Patel AB, et al. Metagenomic analysis of buffalo rumen microbiome: effect of roughage diet on dormancy and sporulation genes. Meta Gene. 2014;2:252-68.

17. Kala A, Kamra DN, Kumar A, Agarwal N, Chaudhary LC, Joshi CG. Impact of levels of total digestible nutrients on microbiome, enzyme profile and degradation of feeds in buffalo rumen. PLoS One. 2017;12:e0172051.

18. Zou C, Gu Q, Zhou X, Zhongsheng X, Muhammad Wl, Tang Q, et al Ruminal microbiota composition associated with ruminal fermentation parameters and milk yield in lactating buffalo in Guangxi, China-A preliminary study. J Anim Physiol Anim Nutr (Berl). 2019:103:1374-9.

19. Wood DE, Lu J, Langmead B. Improved metagenomic analysis with kraken 2. Genome Biol. 2019:20:257. https://doi.org/10.1186/s13059-019-1891-0.

20. Chaumeil P-A, Mussig AJ, Hugenholtz P, Parks DH. GTDB-Tk: a toolkit to classify genomes with the genome taxonomy database. Bioinformatics. 2019. https://doi.org/10.1093/bioinformatics/btz848.

21. Segata N, Izard J, Waldron L, Gevers D, Miropolsky L, Garrett WS, et al. Metagenomic biomarker discovery and explanation. Genome Biol. 2011;12.

22. Franzosa EA, Mclver LJ, Rahnavard G, Thompson LR, Schirmer M, Weingart $\mathrm{G}$, et al. Species-level functional profiling of metagenomes and metatranscriptomes. Nat Methods. 2018;15:962-8.

23. Jovel J, Patterson J, Wang W, Hotte N, O'Keefe S, Mitchel T, et al. Characterization of the gut microbiome using 165 or shotgun metagenomics. Front Microbiol. 2016;7.

24. Kang DD, Li F, Kirton E, Thomas A, Egan R, An H, et al. MetaBAT 2: An adaptive binning algorithm for robust and efficient genome reconstruction from metagenome assemblies. PeerJ. 2019;2019.

25. Stewart RD, Auffret MD, Snelling TJ, Roehe R, Watson M. MAGpy: a reproducible pipeline for the downstream analysis of metagenomeassembled genomes (MAGs). Bioinformatics. 2019;35:2150-2. https://doi.org/ 10.1093/bioinformatics/bty905.

26. Suzek BE, Wang Y, Huang H, McGarvey PB, Wu CH. UniRef clusters: a comprehensive and scalable alternative for improving sequence similarity searches. Bioinformatics. 2015;31:926-32.

27. Seshadri R, Leahy SC, Attwood GT, Teh KH, Lambie SC, Cookson AL, et al. Cultivation and sequencing of rumen microbiome members from the Hungate1000 collection. Nat Biotechnol. 2018;36:359-67.

28. Singh KM, Ahir VB, Tripathi AK, Ramani UV, Sajnani M, Koringa PG, et al. Metagenomic analysis of Surti buffalo (Bubalus bubalis) rumen: a preliminary study. Mol Biol Rep. 2012;39:4841-8.

29. Stewart RD, Auffret MD, Warr A, Walker AW, Roehe R, Watson M. Compendium of 4,941 rumen metagenome-assembled genomes for rumen microbiome biology and enzyme discovery. Nat Biotechnol. 2019;37:953-61.

30. Stewart RD, Auffret MD, Warr A, Wiser AH, Press MO, Langford KW, et al. Assembly of 913 microbial genomes from metagenomic sequencing of the cow rumen. Nat Commun. 2018;9.

31. Matsui H; Ogata K, Tajima K, Nakamura M, Nagamine T, Aminov R, et al. Phenotypic characterization of polysaccharidases produced by four Prevotella type strains. Curr Microbiol 2020;41:45.

32. Stevenson DM, Weimer PJ. Dominance of Prevotella and low abundance of classical ruminal bacterial species in the bovine rumen revealed by relative quantification real-time PCR. Appl Microbiol Biotechnol 2007;75:165-174.

33. Fondevila M, Dehority BA. Interactions between Fibrobacter succinogenes, Prevotella ruminicola, and Ruminococcus flavefaciens in the digestion of cellulose from forages. J Anim Sci 1996;74:678-684.

34. Flint HJ, Bayer EA, Rincon MT, Lamed R, White BA. Polysaccharide utilization by gut bacteria: potential for new insights from genomic analysis. Nat Rev Microbiol. 2008:6:121-31.

35. Vaidya JD, van den Bogert B, Edwards JE, Boekhorst J, van Gastelen S, Saccenti $E_{\text {, et }}$ al. The effect of DNA extraction methods on observed microbial communities from fibrous and liquid rumen fractions of dairy cows. Front Microbiol. 2018;9.

36. Deusch S, Camarinha-Silva A, Conrad J, Beifuss U, Rodehutscord M, Seifert J. A structural and functional elucidation of the rumen microbiome influenced by various diets and microenvironments. Front Microbiol. 2017:8:1605.
37. Avguštin G, Wallace RJ, Flint HJ. Phenotypic diversity among ruminal isolates of Prevotella ruminicola: proposal of Prevotella brevis sp. nov., Prevotella bryantii sp. nov., and Prevotella albensis sp. nov. and redefinition of Prevotella ruminicola. Int J Syst Bacteriol 1997;47:284-288.

38. Wallnöfer P, Baldwin RL. Pathway of propionate formation in Bacteroides ruminicola. J Bacteriol 1967;93:504-505. http://www.ncbi.nlm.nih.gov/ pubmed/6020420. Accessed 12 Feb 2020.

39. Van Nevel CJ, Henderickx HK, Demeyer DI, Martin J. Effect of chloral hydrate on methane and propionic acid in the rumen. Appl Microbiol. 1969;17:695700 http://www.ncbi.nlm.nih.gov/pubmed/5785952. Accessed 12 Feb 2020.

40. Bankevich A, Nurk S, Antipov D, Gurevich AA, Dvorkin M, Kulikov AS, et al. SPAdes: a new genome assembly algorithm and its applications to singlecell sequencing. J Comput Biol. 2012;19:455-77.

41. Seemann T. Prokka: rapid prokaryotic genome annotation. Bioinformatics. 2014;30:2068-9.

42. Steinegger M, Mirdita M, Söding J. Protein-level assembly increases protein sequence recovery from metagenomic samples manyfold. Nat Methods. 2019;16:603-6.

43. Steinegger $M$, Söding J. Clustering huge protein sequence sets in linear time. Nat Commun. 2018;9.

44. Buchfink B, Xie C, Huson DH. Fast and sensitive protein alignment using DIAMOND. Nat Methods. 2014;12:59-60.

\section{Publisher's Note}

Springer Nature remains neutral with regard to jurisdictional claims in published maps and institutional affiliations.

\section{Ready to submit your research? Choose BMC and benefit from:}

- fast, convenient online submission

- thorough peer review by experienced researchers in your field

- rapid publication on acceptance

- support for research data, including large and complex data types

- gold Open Access which fosters wider collaboration and increased citations

- maximum visibility for your research: over $100 \mathrm{M}$ website views per year

At BMC, research is always in progress.

Learn more biomedcentral.com/submissions 